\title{
Systemic cyclosporin A in high risk penetrating keratoplasties: a case-control study
}

A C Poon, J E Forbes, J K G Dart, S Subramaniam, C Bunce, P Madison, L A Ficker, S J Tuft, D S Gartry, R J Buckley

\begin{abstract}
Aim-To examine the efficacy of systemic cyclosporin A (CSA) in preventing rejection and graft failure in high risk keratoplasty (PK).

Methods-A retrospective case-control study with 49 patients in both the CSA group and the control group. The patients receiving CSA were at high risk of graft rejection and failure. Controls were identified from surgical audit books and had high risk characteristics.

Results-There was no statistical difference in preoperative risk factors and the use of postoperative topical steroids between the two groups. The median follow up in the CSA group was 22 months and 27 months in the control group. One or more rejection episodes occurred in 18 out of 49 (36.7\%) cases in the CSA group and 26 out of $49(53.1 \%)$ in the control group. Graft failure from all causes occurred in 16 $(32.7 \%)$ CSA patients and $18(36.7 \%)$ control patients. Four $(8.2 \%)$ of the CSA group compared to eight $(16.3 \%)$ in the control group failed because of rejection. $22(44.9 \%)$ out of 49 patients in the CSA group had side effects. In five (10.2\%) patients, CSA was stopped because of the side effects; eight patients had elevated serum urea and creatinine and four developed hypertension. Minor side effects reported include gum hyperplasia, increased sweating, backache, nausea, feeling unwell, oral candidiasis, cramps, and paraesthesia of the extremities.

Conclusion-These results suggest that the benefit of CSA over conventional therapy in preventing rejection episodes and subsequent graft failure is only moderate and did not reach statistically significant levels in this study. Considering the high frequency of side effects and the cost of CSA, a randomised control trial may be necessary to determine the true value of CSA in high risk penetrating keratoplasty.

(Br f Ophthalmol 2001;85:1464-1469)
\end{abstract}

Cyclosporin A (CSA), a drug with potent immunomodulating activity, is used extensively in organ transplantation to prevent graft rejection. Its low myelotoxicity and specific action on $\mathrm{T}$ cells make it attractive compared with other immunosuppressive drugs. For prophylaxis against corneal graft rejection and failure from rejection, CSA has been used systemically. ${ }^{1-5}$ The use of topical CSA has been reported by a number of authors ${ }^{6-10}$; however, a randomised controlled trial examining the efficacy of topical CSA in preventing rejection and failure in high risk keratoplasty found no beneficial effect over the use of topical steroids. ${ }^{10}$

In a prospective non-randomised trial, Hill presented a case-control study with very encouraging results with systemic CSA, which led to some corneal surgeons using it for high risk penetrating keratoplasty (PK). However, there have been other case-control trials performed with less favourable results. ${ }^{4}{ }^{5} \mathrm{We}$ present the results of a case-control study comparing the results of high risk $\mathrm{PK}$ in patients taking CSA and those not taking CSA at Moorfields Eye Hospital.

\section{Methods}

PATIENTS

At Moorfields Eye Hospital, data on all patients with corneal and external diseases requiring systemic immunosuppression are kept on a database. From February 1992, 49 patients have been prescribed CSA at the time of penetrating keratoplasty (PK) as prophylaxis against allograft rejection. These patients were considered high risk because they had: (1) two or more quadrants of vascularisation, (2) history of ipsilateral graft rejection, (3) active ocular inflammation at the time of PK, or (4) history of chronic intraocular inflammation. Forty nine patients were then identified from the surgical audit books, dating back to 1985, to act as historical controls. Ten control patients had grafts performed before February 1992 and the rest were concurrent with the CSA patients. They were selected if they fulfilled one or more of the high risk criteria listed above and we tried to match for age and disease.

The best corrected or pinhole visual acuity was measured preoperatively and at the last follow up.

SAMPLE SIZE CALCULATION

In order that the study had an $80 \%$ chance of detecting as statistically significant, at the $5 \%$ level, a true reduction in 2 year failure rate from $60 \%$ to $30 \%$ because of CSA usage, 49 cases and 49 controls were recruited into the study (EPI-INFO version 6).

\section{GRAFT MATERIAL CHARACTERISTICS}

Tissue matched grafts were performed in eight CSA patients and nine control patients. The donor cornea was optimally matched for HLA-A and HLA-B. Two or more mismatches were not accepted. Tissue matched donor corneas were preserved in organ culture. 
Table 1 Preoperative factors

\begin{tabular}{|c|c|c|c|c|}
\hline & $\begin{array}{l}\operatorname{CSA}(n=49) \\
\text { No }(\%)\{m\}^{\star}\end{array}$ & $\begin{array}{l}\text { Control }(n=49) \\
\text { No }(\%)\{m\}\end{array}$ & p Value & $\chi^{2}$ Test \\
\hline Male & $32(65)$ & $32(65)$ & & \\
\hline Inflammation at time of operation & $15(31)\{1\}$ & $17(35)$ & 0.72 & \\
\hline Vascularisation $\geqslant 2$ quadrants & $30(61.2)$ & $26(53.1)$ & 0.41 & \\
\hline Previous rejection & 9 & 9 & & \\
\hline \multirow[t]{2}{*}{ HLA matched graft } & $8(16.3)$ & $9(18.4)$ & 0.79 & \\
\hline & Median (IQR) & Median (IQR) & & Rank sum \\
\hline Age (years) & $45(33,52)$ & $58(45,72)$ & $<0.01$ & \\
\hline Number of previous PK & $1(0,1)$ & $0(0,1)$ & 0.30 & \\
\hline Donor size & $8(7.75,8.25)$ & $8(7.75,8.5)$ & & \\
\hline Follow up of clear graft (months) & $22(15,34)$ & $27(12,54)$ & 0.30 & \\
\hline Maximum follow up & 60 & 159 & & \\
\hline
\end{tabular}

${ }^{\star} \mathrm{m}=$ number missing; $\%$ is $\%$ of $\mathrm{n}-\mathrm{m}$.

Table 2 Primary pathology

\begin{tabular}{lll}
\hline Primary pathology & CSA & Control \\
\hline HSV & 3 & 4 \\
Other infections & 10 & 11 \\
Keratoconus and dystrophies & 4 & 7 \\
Chemical burns & 6 & 3 \\
Other inflammatory (RA, atopy, GVH, IK) & 5 & 7 \\
Endothelial failure & 15 & 10 \\
Other (mechanical trauma, aniridia) & 6 & 7
\end{tabular}

HSV $=$ herpes simplex infection; RA = rheumatoid arthritis; $\mathrm{GVH}=$ graft versus host disease; $\mathrm{IK}=$ interstitial keratitis.

Non-matched donor corneas were preserved as corneoscleral discs in culture medium at $4^{\circ} \mathrm{C}$. At Moorfields, McCarey-Kaufman medium was used up to 1991, after which Optisol GS (Chiron Vision) was used. All patients received Optisol GS stored corneas except for six control patients who received MK stored corneas.

\section{TECHNIQUE}

The donor grafts ranged from 6.75 to $11.5 \mathrm{~mm}$ (mean $8.0 \mathrm{~mm}$ ) in the CSA group and 5.0 to 11.5 (mean $8.1 \mathrm{~mm}$ ) in the control group. The grafts were $0.25-0.50 \mathrm{~mm}$ larger than the recipient beds. Eleven CSA patients and 12 controls had simultaneous extracapsular cataract extraction. All had placement of an intraocular lens except for one CSA and three control patients who were left aphakic.

For all patients, 10/0 nylon was used to secure the graft either as interrupted sutures or as one continuous suture. Subconjunctival cefuroxime $(125 \mathrm{mg})$ and betamethasone (4 $\mathrm{mg}$ ) were given at the completion of surgery. Postoperatively, patients were started on topical steroids and chloramphenicol. The frequency of steroid varied from hourly day and night to four times a day. Chloramphenicol drops were instilled four times a day.

Table 3 Postoperative topical and systemic steroid use

\begin{tabular}{llll}
\hline & CSA & Control & p Value \\
\hline Topical steroids for >1 year & 35 out of 36* & 31 out of 36* & $0.20 \ddagger$ \\
No of dropst for the first 3 months (mean) & 455 & 470 & $0.78 ฐ$ \\
No of dropst for the first 6 months (mean) & 756 & 699 & $0.09 \S$ \\
No of dropst for the first 12 months (mean) & 1220 & 1063 & $0.17 \S$ \\
Systemic steroids during follow up period & 21 out of 49 & 10 out of 49 & $0.03 \ddagger$ \\
\hline
\end{tabular}

${ }^{\star}$ Out of those with at least 1 year follow up and have not failed.

†Dexamethasone equivalent drops. Non-dexamethasone drops converted to equivalent dexamethasone drops using the following relative potencies. Dexamethasone alcohol $0.1 \%=1$; prednisolone acetate $1 \%=1.275$; fluoromethalone alcohol $0.1 \%=0.775$; prednisolone phosphate $0.3 \%=0.35 .{ }^{11}$

$\ddagger$ Fisher exact test.

$§$ Mann-Whitney test.
CSA THERAPY

Patients receiving CSA underwent full blood and metabolic analysis before CSA was started. CSA was initiated at the time of surgery or just before. The preparation of CSA used was changed from Sandimmun (Novartis) to Neoral (Sandoz) in 1997. The dose used was between 3 and $5 \mathrm{mg} / \mathrm{kg}$ per day. Monthly trough serum CSA levels were performed and the dosages adjusted so that the trough levels fell between 75 and $180 \mu \mathrm{g} / \mathrm{l}$. The serum CSA is measured using the EMIT 2000 cyclosporin specific assay (Behring), which is based on monoclonal antibodies. Baseline and monthly blood pressure measurements were performed together with renal function tests, liver function tests, and a full blood count. CSA was used for at least 12 months unless side effects required its cessation. All recorded side effects were noted with CSA therapy.

\section{REJECTION CRITERIA AND TREATMENT OF} REJECTION

Rejection was defined as the occurrence of one of the following: (1) development of a rejection line (epithelial or endothelial); (2) new unilateral anterior chamber reaction with $\mathrm{KP}$; (3) increasing corneal oedema in a previously clear compact graft with visible aqueous cells.

Rejection episodes were treated with hourly dexamethasone $0.1 \%$ drops day and night and subconjunctival betamethasone $(4 \mathrm{mg})$ was given in the majority of cases. The dexamethasone was weaned in accordance to the clinical response. Some patients received $500 \mathrm{mg}$ intravenous methylprednisolone for rejection episodes.

Graft failure was defined as the permanent loss of corneal transparency in a previously thin and clear cornea. Graft failure secondary to rejection was defined as when failure was thought to be due primarily to rejection.

ASSESSMENT OF TOPICAL STEROID AND SYSTEMIC STEROID USE

The type, frequency, and duration of topical steroids used postoperatively were recorded for all patients. Leibowitz presented data on relative potency of various topical steroid formulations, based on inhibition of polymorphonuclear leucocyte migration into the cornea in the setting of keratitis. ${ }^{11}$ Using these data we converted the topical medications used into dexamethasone equivalent units and were able 
to compare the steroid usage between the patients of the two groups. All patients except for one CSA patient and three control patients were started on dexamethasone as the initial postoperative topical steroid. Of the four patients not started on dexamethasone immediately postoperatively, the patient in the CSA

Table 4 Rejection and failure rates: overall ${ }^{\star}$, and at 1 and 2 year follow up

\begin{tabular}{|c|c|c|c|}
\hline & $C S A$ & Control & Fisher \\
\hline \multicolumn{4}{|l|}{ Rejection } \\
\hline Overall* & $18 / 49(36.7 \%)$ & $26 / 49(53.1 \%)$ & $\mathrm{p}=0.16$ \\
\hline 1 year & $11 / 45(24.4 \%)$ & $14 / 43(32.6 \%)$ & $\mathrm{p}=0.48$ \\
\hline 2 years & $15 / 33(45.5 \%)$ & $21 / 37(56.8 \%)$ & $\mathrm{p}=0.47$ \\
\hline \multicolumn{4}{|c|}{ Failure (all causes) } \\
\hline Overall* & $14 / 49(28.6 \%)$ & $18 / 49(36.7 \%)$ & $\mathrm{p}=0.71$ \\
\hline 1 year & $6 / 45(13.3 \%)$ & $5 / 43(11.6 \%)$ & $\mathrm{p}=1.00$ \\
\hline 2 years & $11 / 33(33.3 \%)$ & $8 / 37(21.6 \%)$ & $\mathrm{p}=0.30$ \\
\hline \multicolumn{4}{|c|}{ Failure (rejection) } \\
\hline Overall* & $4 / 49(8.2 \%)$ & $8 / 49(16.3 \%)$ & $\mathrm{p}=0.36$ \\
\hline 2 years & $1 / 33(3.0 \%)$ & $3 / 37(8.1 \%)$ & $\mathrm{p}=0.62$ \\
\hline
\end{tabular}

${ }^{\star}$ Overall rates do not take into account the shorter overall follow up in the CSA group.

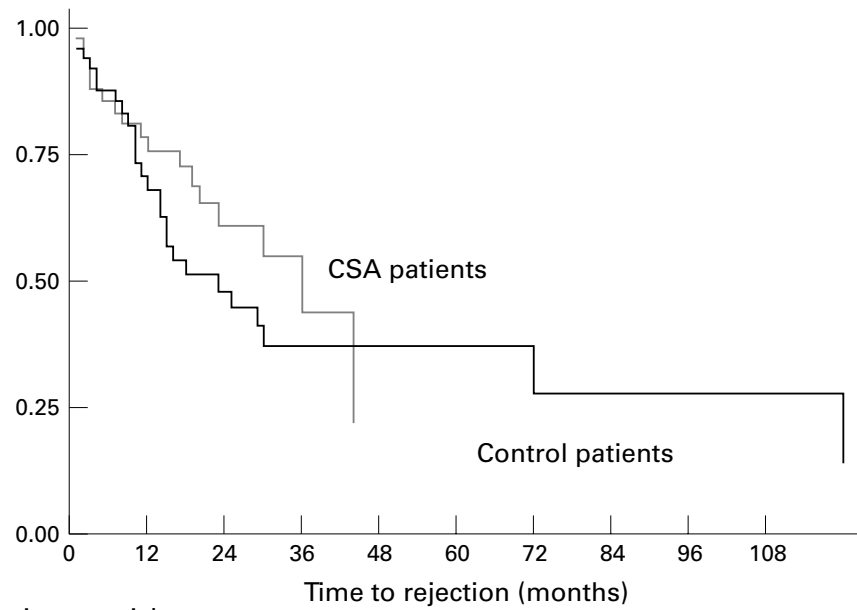

No of patients at risk

$\begin{array}{lllllll}\text { Control } & 49 & 27 & 15 & 9 & 8 & 4 \\ \text { CSA } & 49 & 28 & 13 & 5 & 0 & \end{array}$

Figure 1 Kaplan-Meier survival estimates for rejection. Difference between the CSA and control groups was not significant when compared by log rank and Wilcoxon (Breslow) tests. The number of study patients at risk at various points after time zero are shown below the horizontal axis.

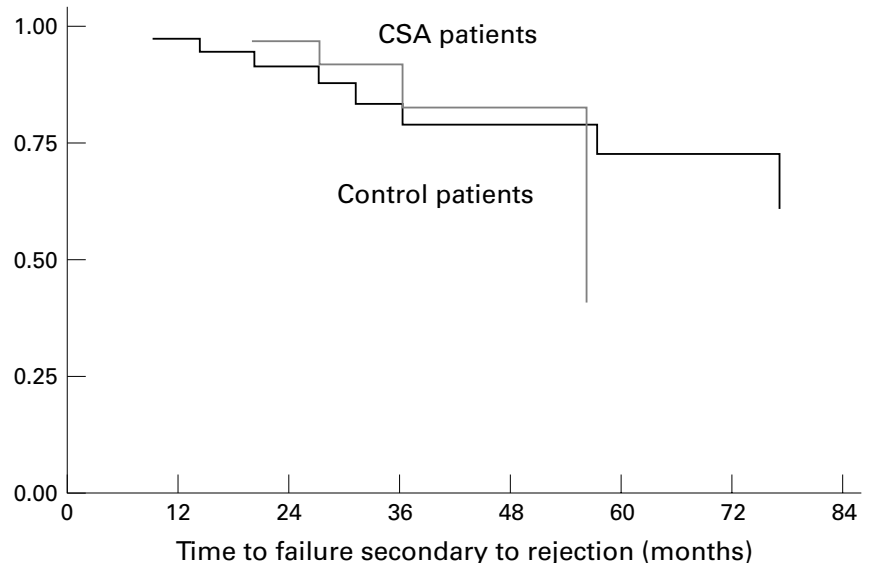

No of patients at risk

$\begin{array}{lrrrrrr}\text { Control } & 49 & 37 & 27 & 19 & 15 & 10 \\ \text { CSA } & 49 & 38 & 22 & 10 & 3 & 1\end{array}$

Figure 2 Kaplan-Meier survival estimates for failure from rejection. Difference between the CSA and control groups was not significant when compared by log rank and Wilcoxon (Breslow) tests. The number of study patients at risk at various points after time zero are shown below the horizontal axis. group was taking prednisolone phosphate $0.3 \%$ and the three control patients were taking prednisolone acetate $1 \%$.

\section{STATISTICS}

The age, sex, and various preoperative risk factors were compared between the cases and controls (Table 1). $\chi^{2}$ tests were used to compare categorical variables and the rank sum test was used to compare continuous variables.

Kaplan-Meier plots of the estimated probabilities over time, of rejection, failure and failure due to rejection were constructed and "survival" experiences compared by the log rank test. The proportion of rejections, failures and failures from rejection at 1 and 2 years were compared using Fisher's exact test.

\section{Results}

PATIENTS

Table 1 shows that the median age of the CSA group was significantly less than that of the control group $(p<0.01)$. This was due to the reluctance to use CSA in elderly patients. The follow up periods of the two groups are also different, but not statistically significantly so. Table 2 compares the primary corneal pathology requiring grafting to be performed in the two groups.

TOPICAL AND SYSTEMIC STEROID USE

Table 3 compares the steroid use between the patients of the two groups. There was no statistically significant difference between the mean number of dexamethasone equivalent drops taken per patient at 3, 6, and 12 months postoperatively. There was no significant difference in the number of patients receiving topical steroids for more than 1 year. However, there were a greater number of CSA patients requiring systemic prednisolone postoperatively. Four patients in the CSA group and three in the control group received $500 \mathrm{mg}$ intravenous methylprednisolone for rejection episodes. One control patient had oral prednisolone (1 $\mathrm{mg} / \mathrm{kg}$ ) weaned over 2 months for a rejection episode. The other patients requiring systemic steroids took oral prednisolone for significant ocular surface or intraocular inflammation (for example, atopic sclerokeratitis, scleritis, and episcleritis related to infections, and rheumatoid related sclerokeratitis).

\section{REJECTION AND FAILURE RATES}

One and 2 year rates of rejection, failure from all causes, and failure from rejection of the two groups are compared in Table 4 . In all groups, no significant difference was found between the CSA and control groups. Figures 1, 2, and 3 are the Kaplan-Meier survival plots for rejection, failure from rejection, and failure from all causes. Log rank testing revealed no statistical significance between the two groups for the three outcomes. It should be noted, however, that power to detect differences in survival experiences is related to the number of events rather than the number of patients, and is therefore low. 


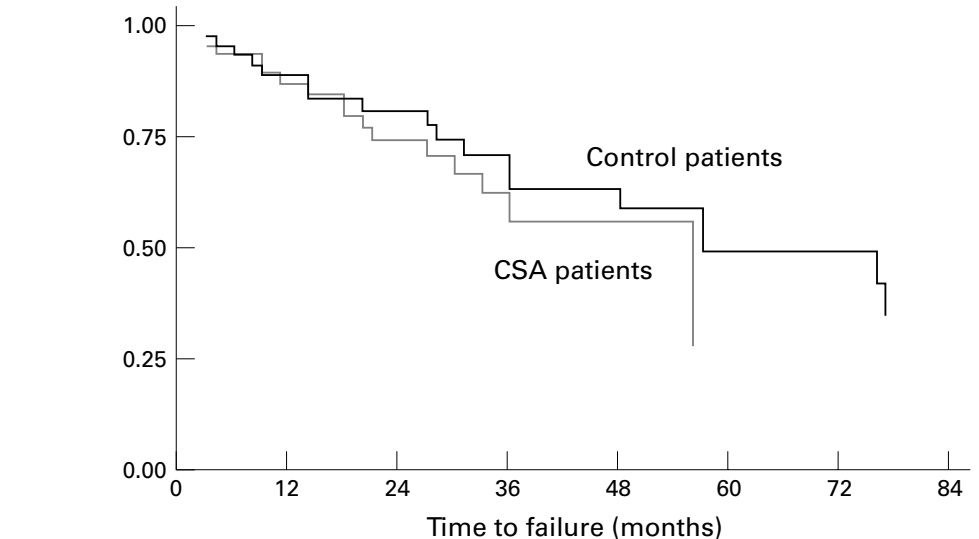

No of patients at risk

$\begin{array}{lrrrrrr}\text { Control } & 49 & 37 & 27 & 19 & 15 & 10 \\ \text { CSA } & 49 & 38 & 22 & 10 & 3 & 1\end{array}$

Figure 3 Kaplan-Meier survival estimates for failure (all causes). Difference between the CSA and control group was not significant when compared by log rank and Wilcoxon (Breslow) tests. The number of study patients at risk at various points after time zero are shown below the horizontal axis.

Table 5 Side effects recorded

\begin{tabular}{ll}
\hline Side effect & No \\
\hline Increased creatinine and urea & 8 \\
Paraesthesia & 3 \\
Hypertension & 4 \\
Felt unwell & 4 \\
Nausea and vomiting & 3 \\
Elevated liver enzymes & 2 \\
Backache & 2 \\
Gingival hyperplasia & 1 \\
Sweating & 1 \\
Cramps & 1 \\
Diarrhoea & 1 \\
Shakes & 1 \\
Oral candidiasis & 1 \\
\hline
\end{tabular}

Of the 26 patients in the control group who rejected, their median time to rejection was 11.5 months. By comparison, the 18 patients in the CSA group rejected at a median of 9.5 months.

Twelve of the $18(24.5 \%)$ rejected while on CSA at a median of 4 months (range 1-17 months). Six grafts rejected after CSA had been stopped for a median of 18 months (range 5-28 months). The median duration of CSA use for the six grafts was 13.5 months.

SIDE EFFECTS

Table 5 lists the side effects and their frequency. Twenty two of the 49 patients $(44.9 \%)$ on CSA had recorded side effects. Five patients (10.2\%) stopped CSA because of side effects. A further nine patients required reduction in CSA.

VISUAL ACUITY

Figures 4 and 5 demonstrate that patients in both groups tended to improve postoperatively. Twenty one of the $49(42.9 \%)$ CSA patients and 13 out of the 49 (26.5\%) control patients had a corrected or pinhole VA of $6 / 18$ or better $(p=0.09)$ at last postoperative visit.

\section{Discussion}

In this study, cases and controls were similarthe only statistically significant difference being age and the use of systemic steroids in the CSA

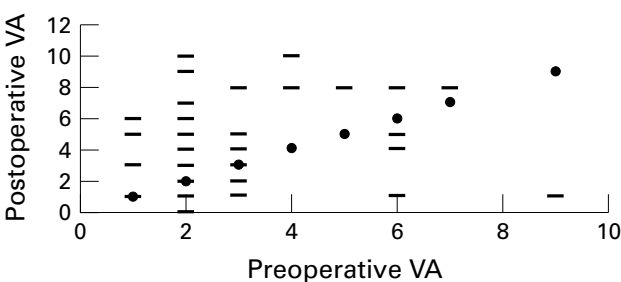

Figure 4 Visual acuity (corrected or with pinhole) of control patients, preoperative versus postoperative acuity. VA units: $0=$ no perception of light, $1=$ perception of light, $2=$ hand movement, $3=$ counting fingers, $4=6 / 60,5=$ $6 / 36,6=6 / 24,7=6 / 18,8=6 / 12,9=6 / 9,10=6 / 6$.

Solid circle represents the "no change"line where the postoperative acuity equals preoperative acuity; bar represents the postoperative VA plotted against the preoperative VA. Those above the line of "no change" represent an improvement of VA.

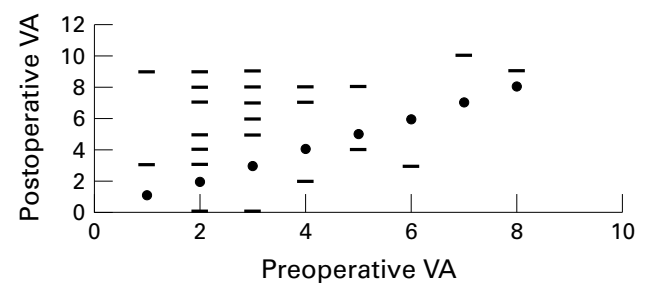

Figure 5 Visual acuity (corrected or with pinhole) for CSA patients, preoperative versus postoperative VA. VA units: $0=$ no perception of light, $1=$ perception of light, $2=$ hand movement, $3=$ counting fingers, $4=6 / 60,5=6 / 36$, $6=6 / 24,7=6 / 18,8=6 / 12,9=6 / 9,10=6 / 6$. Solid circle represents the "no change" line where the postoperative acuity equals preoperative acuity; bar represents the postoperative VA plotted against the preoperative VA. Those above the line of "no change" represent an improvement of VA.

group. The relatively small difference in age is unlikely to have influenced the results; Williams et al looked at the influence of advanced recipient age on outcome of corneal transplantation and found that graft survival falls significantly in recipients aged 60 or more years when compared with outcome in younger patients. However, they concluded that the different indications for grafting between the young and the old recipients account for most if not all of the difference in graft survival and advanced age in itself does not have a major impact on graft survival. ${ }^{12}$ The difference in systemic steroid use is one of the weaknesses of a retrospective study design. The reasons for this are uncertain but include a change in practice between 1985, when data collection started for the control patients, and 1992 when data collection started for the CSA patients. Clinicians, already more comfortable with using systemic CSA, may have been less concerned about the addition of systemic steroids than they were before 1992 when topical therapy alone was more usual. Alternatively, the CSA patient group may have been more subject to episodes of inflammation than the control group despite having similar disorders. However, the CSA group had more patients with chemical injuries while the control group had more in the keratoconus and dystrophy category, suggesting that the CSA group may have been at higher risk of rejection. However, these conditions were the initial corneal pathology and all eyes had high risk characteristics by the time of the PK in question. 
Table 6 Comparison with other case/control studies

\begin{tabular}{|c|c|c|c|c|c|c|}
\hline & \multicolumn{3}{|c|}{ Rejection } & \multicolumn{3}{|c|}{ Failure from rejection } \\
\hline & \multirow{2}{*}{$\begin{array}{l}C S A \\
37 \%\end{array}$} & \multicolumn{2}{|c|}{ Control } & \multirow{2}{*}{$\begin{array}{l}C S A \\
8 \%\end{array}$} & \multicolumn{2}{|c|}{ Control } \\
\hline Poon, $n=49$ & & $53 \%$ & ns & & $16 \%$ & $\mathrm{~ns}$ \\
\hline Hill $^{1-2}, \mathrm{n}=43$ & $49 \%$ & $73 \%$ & sig & $23 \%$ & $62 \%$ & sig \\
\hline Robert $^{4}, \mathrm{n}=45$ & $70 \%$ & $50 \%$ & ns & $50 \%$ & $40 \%$ & ns \\
\hline Volker-Dieben $^{5}, n=21$ & & & & $12 \%$ & $15 \%$ & ns \\
\hline
\end{tabular}

$\mathrm{ns}=$ not significant difference. sig = significant difference.

In this study, donor material was stored in McCarey-Kaufman solution, Optisol GS, or tissue culture. There are various published comparisons between the three storage methods, ${ }^{13-16}$ and no difference has been found in graft outcome.

This study showed trends towards reduced rates of rejection and failure from rejection in high risk PK with systemic immunosuppression with CSA. Failure from all causes, however, appeared to occur more rapidly in the CSA patients than the controls. However, the differences between the CSA and the control groups did not reach statistical significance. The rates of rejection and failure reported here are lower than that found by Hill, ${ }^{12}$ perhaps because his patients had different risk factors than those in this study. Hill's patients all had three to four quadrants of corneal vascularisation, while in this study, 30 and 26 patients in the CSA and control groups respectively had two or more quadrants of vascularisation and other patients had risk factors such as previous rejection episodes or inflammation at the time of surgery. In addition Hill's use of postoperative steroids was less intense and of shorter duration compared to this study; his patients received dexamethasone $0.1 \%$ drops four times daily and betamethasone $0.1 \%$ ointment at night initially and the drops were slowly weaned and stopped at 6 months postoperatively. By comparison, 35 of the CSA patients and 31 of the control patients were still using topical steroids at 1 year in this study. At 6 months the mean dexamethasone equivalent drops used was 756 and 699 in the CSA and control drops respectively; that is equivalent to approximately four drops daily for the whole of the 6 months in both groups.

There are now three case/control series showing CSA does not significantly decrease the rate of rejection or failure due to rejection. The study by Robert et al found that CSA did not make any difference in the rate of rejection or failure. ${ }^{4}$ CSA was administered at a dose of $5 \mathrm{mg} / \mathrm{kg} /$ day and trough levels were kept at 100-150 ng/1 for 3 months. They attributed the results to insufficient dose and duration of CSA, and inadequately matched patients. Volker-Dieben et al failed to show any difference in failure from rejection, ${ }^{5}$ but as all the grafts were HLA matched it is possible that an effect of CSA may have been masked (see Table 6).

Various groups have reported on the use of topical CSA..$^{6-9}$ However, a randomised controlled study sponsored by Sandoz failed to demonstrate improved graft survival with topical CSA. ${ }^{10}$ Allosensitisation following a corneal graft may take place outside the eye, at the local lymph nodes and this may account for the lack of effect by topical therapy.

Forty five per cent of the patients in this study had recorded side effects. Other groups have reported side effects in $29-38 \%$ of patients. $^{2417}$ Ten per cent of patients stopped because of side effects. All side effects were reversible after cessation of CSA. The use of CSA in solid organ transplantation has been associated with an increased risk of lymphoproliferative disorders that has been attributed to the long duration of therapy and the use in conjunction with other immunosuppressive drugs. ${ }^{18}$ The mechanism has been thought to be secondary to the impairment of the organ recipient's immune surveillance system, but there is now evidence that cyclosporin has a direct stimulatory effect on malignant cells via a TGF- $\beta$ related mechanism. ${ }^{19}$ With this new evidence suggesting CSA has a direct role in progression of malignant disease, we may need to reconsider our indications for use with eye conditions.

This study supports the proposition that CSA may well have some effect on survival of high risk corneal grafts. However, this effect, like that in two of the other case controlled studies, ${ }^{45}$ was not significant unlike the result of Hill's study. This may be because of the predominance of corneal vascularisation as a risk factor in Hill's study compared to the present study. Corneas that are vascularised are not necessarily at higher risk of rejection compared to a non-vascularised cornea that has rejected three or four times. It may be that the effect of CSA on rejection in vascularised corneas is different from that in corneas with previous rejection episodes and inflammation as risk factors. Another possible explanation why CSA did not improve graft survival is that the effect of CSA may have been drowned by the intensive use of topical steroids. Topical steroid was used more frequently and for longer periods in our study compared with the other case-control studies. ${ }^{1245}$ Long term topical steroid use may well be effective as systemic immunosuppression in high risk keratoplasty. In patients who are pseudophakic or aphakic, and who do not suffer from a rise in IOP from use of steroids, long term steroids may be a very safe option. The cost of CSA taken at a dose of $150 \mathrm{mg}$ twice daily is $£ 3200$ per year in the United Kingdom. Considering the high frequency of side effects and the cost of CSA, a randomised control trial may be necessary to determine the true value of CSA in high risk keratoplasty. Power calculations, using Pocock's formula, indicate that over 200 patients would be needed in both groups to show a reduction in failure from rejection in 5 years from $40 \%$ to $24 \%$ at $5 \%$ significance and $80 \%$ power, allowing for a $10 \%$ loss to follow up. This study would need to compare the efficacy of CSA with the use of long term topical steroids. In addition, there are a number of newer immunosuppressants, like mycophenolate mofetil and tacrolimus, which may be useful for prophylaxis against corneal graft rejection and failure. 
1 Hill JC. Systemic cyclosporine in high-risk keratoplasty: long term results. Eye 1995;9:422-8.

2 Hill JC. Systemic cyclosporine in high-risk keratoplasty: short versus long-term therapy. Ophthalmology 1994;101: 128-33.

3 Sundmacher R, Reinhard T, Heering P. Six years' experience with systemic cyclosporin A prophylaxis in high-risk perforating keratoplasty patients: a retrospective study. Ger f Ophthalmol 1992;1:432-6.

4 Robert PY, Delbosc B, Preux PM, et al. Traitement par ciclosporine A, a doses faibles, dans les keratoplasties transfixiantes a haut risque. Etude bicentrique de 90 cas. $\mathcal{F}$ Fr Ophtalmol 1997;20:507-14.

5 Volker-Dieben HJ, D'Amaro J, Van Slooten H, et al. The effect of cyclosporin A on corneal graft survival. In: Zieruth $\mathrm{M}$, ed. 9th ed. Immunology of corneal transplantation. Buren: Aeolus Press, 1995:239-46.

6 Belin MW, Bouchard CS, Frantz S, et al. Topical cyclosporine in high-risk corneal transplants. Ophthalmolcyclosporine in high-

7 Goichot-Bonnat L, Chemla P, Pouliquen Y. Topical cyclosporine A to prevent the corneal rejection. $\mathcal{f} F r$ Ophtalmol 1987:10:213-17.

8 Hoffmann F, Wiederholdt M. Local treatment of corneal transplant in humans with cyclosporin A. Klin Monatsbl Augenheilkd 1985;187:92-6.

9 Holland EJ, Olsen TW, Ketcham JM, et al. Topical cyclosporin A in the treatment of anterior segment inflammatory disease. Cornea 1993;12:413-19.
10 Bouchard CS, Cavanagh HD. The high-risk keratoplasty patient-quo vadis? Cornea 1994;13:1-4.

1 Leibowitz HM. Manaement of inflammation in the cornea and conjunctiva. Ophthalmology 1980;87:753-8.

12 Williams KA, Muehlberg SM, Lewis RF, et al. Influence of advanced recipient and donor age on the outcome of corneal transplantation. Br f Ophthalmol 1997;81:835-9.

13 Frueh BE, Bohnke M. Prospective, randomized clinical evaluation of optisol vs organ culture corneal storage media. Arch Ophthalmol 2000;118:757-60.

14 Lindstrom RL, Kaufman HE, Skelnik DL, et al. Optisol corneal storage medium. Am f Ophthalmol 1992;114:345-56.

15 Bourne WM, Lindstrom RL, Doughman DJ. Endothelial cell survival on transplanted human corneas preserved by organ culture with $1.35 \%$ chondroitin sulfate. $\mathrm{Am} \not{7}$ Ophthalmol 1985;100:789-93.

16 Rijneveld WJ, Beekhuis HW, van Rij, et al. Clinical comparison of grafts stored in McCarey-Kaufman medium at $4^{\circ} \mathrm{C}$ and in corneal organ culture at $31^{\circ} \mathrm{C}$. Arch Ophthalmol 1992:110:203-5.

17 Lim L, Cheong J, Vathsala A, et al. Reducing systemic complications in cyclosporin treatment for high risk keratoplications in cyclosporin treatment for high

18 Boubenider S, Hiesse C, Goupy C, et al. Incidence and consequences of post-transplantation lymphoproliferative disorders. F Nephrol 1997;10:136-45.

19 Hojo M, Morimoto T, Maluccio M, et al. Cyclosporine induces cancer progression by a cell-autonomous mechanism. Nature 1999;397:520-34.

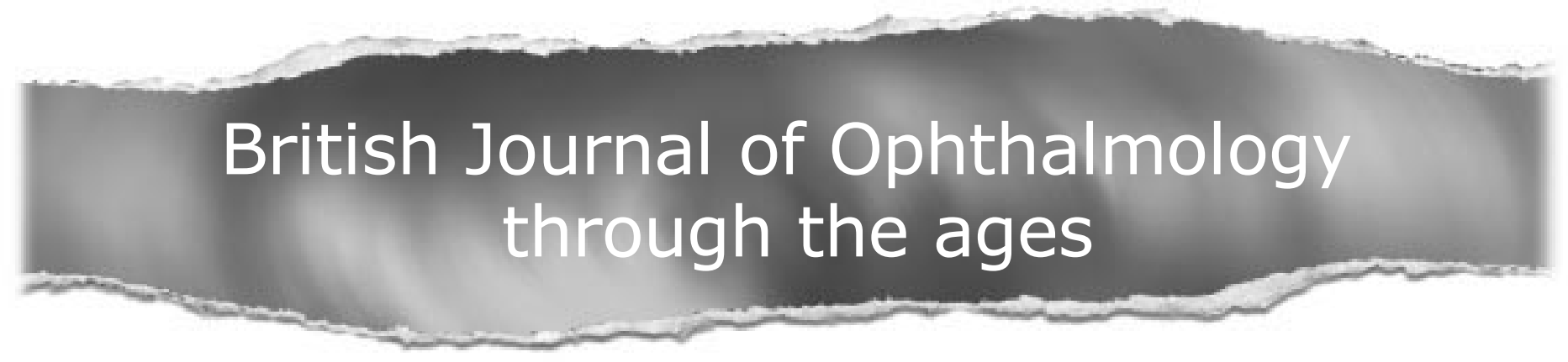

Browse the Archive

British Journal of Ophthalmology online has an archive of content dating back to 1965.

Full text from 1997; abstracts from 1975; table of contents from 1965

www.bjophthalmol.com 